\title{
Proceedings of the 8th International Workshop on Oceanography and Fisheries Science of the East China Sea
}

\author{
James Davis Reimer · Shoichiro Suda
}

Received: 20 July 2012/Revised: 20 July 2012/ Accepted: 20 July 2012/Published online: 19 August 2012

(C) The Oceanographic Society of Japan and Springer 2012

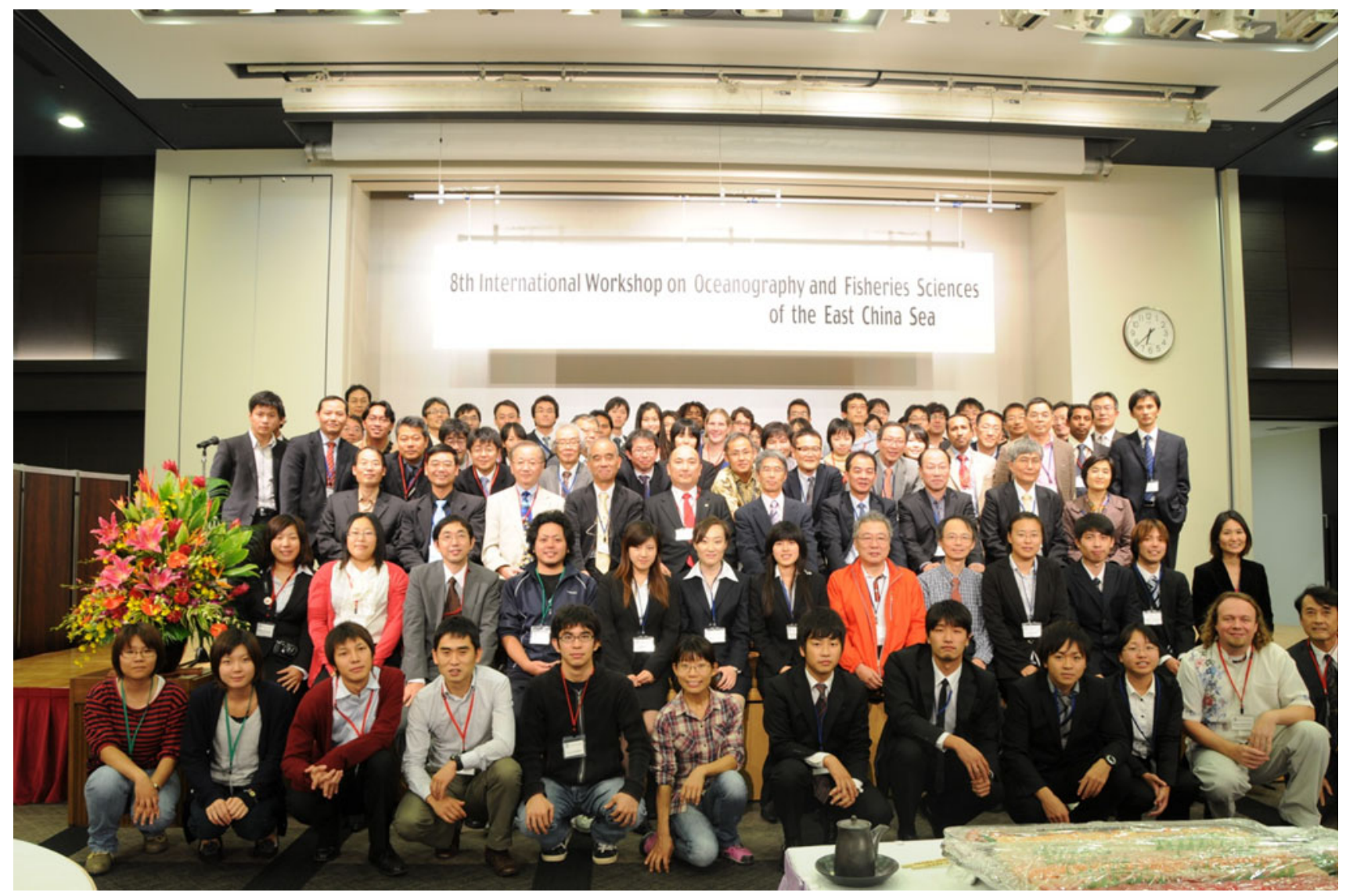

J. D. Reimer $(\bowtie)$

Molecular Invertebrate Systematics and Ecology Laboratory, Rising Star Program, Trans-disciplinary Organization

for Subtropical Island Studies, University of the Ryukyus,

1 Senbaru, Nishihara, Okinawa 903-0213, Japan

e-mail: jreimer@sci.u-ryukyu.ac.jp

\section{S. Suda}

Department of Chemistry, Biology and Marine Science,

Faculty of Science, University of the Ryukyus,

1 Senbaru, Nishihara, Okinawa 903-0213, Japan
This special issue of the Journal of Oceanography is dedicated to the proceedings of the 8th International Workshop on Oceanography and Fisheries Science of the East China Sea (ECSW8), which was held on November 24 and 25, 2011 in Naha, Okinawa, Japan. The ECSW was started in 1997 by four institutions, Shanghai Ocean University (China), Jeju University (Korea), Nagasaki University, and University of the Ryukyus (Japan), as a meeting held every 3 years to promote the exchange of results and ideas related 
to the shared region of the East China Sea. For ECSW8, a fifth institution, National Taiwan Ocean University (Taiwan) was welcomed into the group for the first time.

These five institutions all share the East China Sea as a border. Often relatively overlooked when compared to other oceanic regions such as the Mediterranean, the regions bordering the East China Sea total over 1.5 billion people, or more than $22 \%$ of the total global population. Evidence of the importance of the East China Sea can be seen in tensions over Exclusive Economic Zones (EEZs) in this region. Given the rapid development of China, and the well-established economies of Japan, Korea, and Taiwan, our understanding and research on the East China Sea is not only of regional but truly global importance. At the same time, the East China Sea is important as a diverse and unique oceanic region. Although most of the East China Sea consists of coastal continental shelves and temperate waters, it also includes deep-sea back arcs with chemosynthetic ecosystems, and coral reef regions with some of the highest biodiversity on Earth. Clearly, effective communication and cooperation underpinned by high levels of research will help all countries sharing the East China Sea region further develop their economies in a responsible and sustainable manner while protecting the unique biodiversity of the region for future generations.

In total there were 77 presentations at ECSW8, consisting of 38 oral presentations and 39 poster presentations, and over 120 participants, making ECSW8 the largest ECSW held to date. Given the increasing scientific and geopolitical interest in the East China Sea, it is highly likely that future workshops will continue to see an increase in participation. The oral presentations were divided into eight different sessions, covering topics ranging from the impact of climate change and ocean acidification to fisheries biology, ocean sciences, and calcification of marine organisms. Of these eight sessions, three were specifically for students, boding well for the future of East China Sea research. Additionally, there was a special session devoted to the future of education and double degrees in participating institutions.

The contents of this special issue include five original papers, and they reflect the diversity of the ecosystems of the East China Sea, as well as the diversity of participants in ECSW8. Topics examined include the mangrove areas of Okinawa, ecological data on coral reef fishes, and the characterization of dead zone sediments in coastal bays. We sincerely hope that these manuscripts, as well as all of the other excellent presentations of ECSW8, continue to spur and advance the understanding of our shared border, the East China Sea.

Acknowledgments We sincerely thank all authors and reviewers for their support in helping make this special issue a success. We could not have made this special issue without their hard work and feedback. As well, we wish to thank the ECSW8 organizing committee at the University of the Ryukyus (UR), including Makoto Yamazato, Yukiharu Hisaki, Makoto Tsuchiya, Akihiro Takemura, Yuzuru Ikeda, Hiroyuki Fujimura, Katsunori Tachihara, Hideyuki Imai, and Haruko Kurihara. Harumi Kameshima, Mariko Gibu, and Mayuko Oyakawa aided in organizing and managing all the paperwork and arrangements. Many UR students volunteered their time to help make the workshop run smoothly. Okinawa Churaumi Aquarium generously helped with the organization of the field trip. ECSW8 was supported in part by the International Graduate Program for the AsiaPacific Region, the International Research Hub Project for Climate Change and Coral Reef/Island Dynamics, and the University of the Ryukyus Foundation, all at UR. 